\title{
Herpes simplex type-1 pneumonia in an immunocompetent patient: a case report
}

\author{
Sara Camões ${ }^{1}$, Filipa Lima ${ }^{2}$, Alexandre Vasconcelos ${ }^{1}$ \\ ${ }^{1} /$ nternal Medicine Service, Hospital Pedro Hispano, Matosinhos, Portugal. \\ ${ }^{2}$ Pneumology Service, Centro Hospitalar Vila Nova de Gaia/Espinho, Vila Nova de Gaia, Portugal
}

\begin{abstract}
Herpes simplex virus type-1 pneumonia is unusual and rarely described without any degree of immunosuppression. We share a case of herpes simplex virus type-1 pneumonia in an immunocompetent patient, not only by its rarity, but to call attention to the importance of thinking about this entity when respiratory symptoms persist despite various antibiotic schemes, especially in the presence of ground glass or multifocal pulmonary infiltrates, regardless of patients' immune status.

Keywords: Herpes Simplex, Viral Pneumonia, Immunity.
\end{abstract}

\section{Introduction}

Herpes simplex virus type-1 (HSV-1) infection is extremely prevalent, as more than $90 \%$ of the world's population has positive serological tests by the fourth decade of life. ${ }^{1}$ Virus transmission occurs from person-to-person mainly by contact with infected oropharyngeal lesions, with an incubation period ranging from 1 to 26 days (median 6 to 8 days). ${ }^{1,2}$ Gingivostomatitis and pharyngitis are the most common manifestations of primary infection and labial herpes the most frequent form of reactivation. HSV-1 typically causes recurrent infections and similar to what happens with herpes zoster, the reactivation could occur through the lymph nodes where the virus is latent, in the absence of oropharyngeal lesions. ${ }^{3}$

HSV-1 pneumonia is unusual, but may arise in primary or recurrent infection. There are case reports in burned, transplanted and cancer patients, people under corticosteroids ${ }^{4}$, mechanically ventilated, malnourished or infected with human immunodeficiency virus (HIV), but HSV-1 pneumonia is rarely described without any degree of immunosuppression., ${ }^{1,5}$

\section{Clinical case}

We present a 50-year-old Caucasian non smoker man, with no relevant background. Referred to the Emergency Room with asthenia, anorexia, fever $\left(40^{\circ} \mathrm{C}\right)$, myalgia and productive cough with a week of evolution, already treated with levofloxacin for 3 days without improvement. He had visited a farm about 5 days before the onset of symptoms, but denied consumption of fresh farm cheese or well water. On the physical examination he had rhonchi scattered on auscultation, analytically a reactive $C$ protein of $242,6 \mathrm{mg} / \mathrm{L}$ without leukocytosis, with normal renal function. Chest $\mathrm{X}$-ray showed diffuse pulmonary infiltrates (Fig. 1) with an arterial blood gas showing hypoxemia (p02 $55 \mathrm{mmHg}$ ). Assuming community-acquired pneumonia, he started empirically ceftriaxone and azithromycin but had an unfavorable evolution, maintaining persistent fever and hypoxemia on the fourth day of treatment. We were unable to identify an agent in the screening initially performed (blood cultures, urinary antigen test for Streptococcus pneumoniae and Legionella pneumophila, nasopharyngeal aspirate for influenza virus, bacteriological and mycobacterial sputum culture). Because of the context for zoonotic disease and poor response to the previous antibiotherapy, treatment was changed to doxycycline but with no further response. Chest tomography showed multiple interstitial opacities scattered with ground glass areas, most exuberant in the lung bases (Fig. 2A and B), opening a range of differential diagnoses initially not placed. The immune study was nonspecific, showing only positive antinuclear antibodies (1/160 mottled pattern) with normal immunoglobulins and complement, without anti-neutrophil cytoplasmic antibodies or rheumatoid factor. The serological tests for HIV, Mycoplasma pneumoniae, Chlamydophila pneumoniae, Chlamydophila psittaci and Coxiella burnetii, were negative. Chasing the diagnosis, we performed a bronchofibroscopy that showed whitish micronodules scattered through the trachea and bronchial tree, with mucosal hyperemia; bronchial and bronchoalveolar lavage were obtained and sent to microbiological and anatomopathological analysis. Cytology was negative for malignant cells. On the eighth day of hospitalization, HSV-1 was identified by polymerase chain reaction (PCR) in bronchial lavage, with negative PCR for herpes simplex type-2 and cytomegalovirus. With this information we suspended antibiotics and began intravenous acyclovir monotherapy, $10 \mathrm{mg} / \mathrm{kg}$ three times a day for 10 days, with rapid clinical and analytical improvement. Reviewing the case, the patient didn't have oral lesions and he was never under corticosteroids. Cytology was revised with the knowledge of a positive PCR for HSV-1, but no viral inclusions were found.

\section{Discussion}

The diagnosis of HSV-1 infection was classically based on the presence of cytological abnormalities, particularly the presence of cytoplasmic inclusions and homogenization of nuclear chromatin ${ }^{2,6}$, but to observe these abnormalities the sample must be immediately transported to the laboratory and stored at $4^{\circ} \mathrm{C}$ under the penalty of not being able to observe the viral cytopathic effect due to technical reasons ${ }^{1}$, possibly what happened in our case report. Molecular biology techniques, such as PCR, have gained increasing importance as they overcome cytology limitations. Despite its undeniable value, the relevance of a positive PCR for HSV-1 in critically ill patients, especially those with acute respiratory distress under mechanical ventilation, is unclear. ${ }^{7,8}$

In the context of pneumonia refractory to standard antimicrobial therapy, the possibility of HSV pneumonia must be pursued ${ }^{9}$, 
Fig. 1. Chest X-ray showing multifocal consolidations.

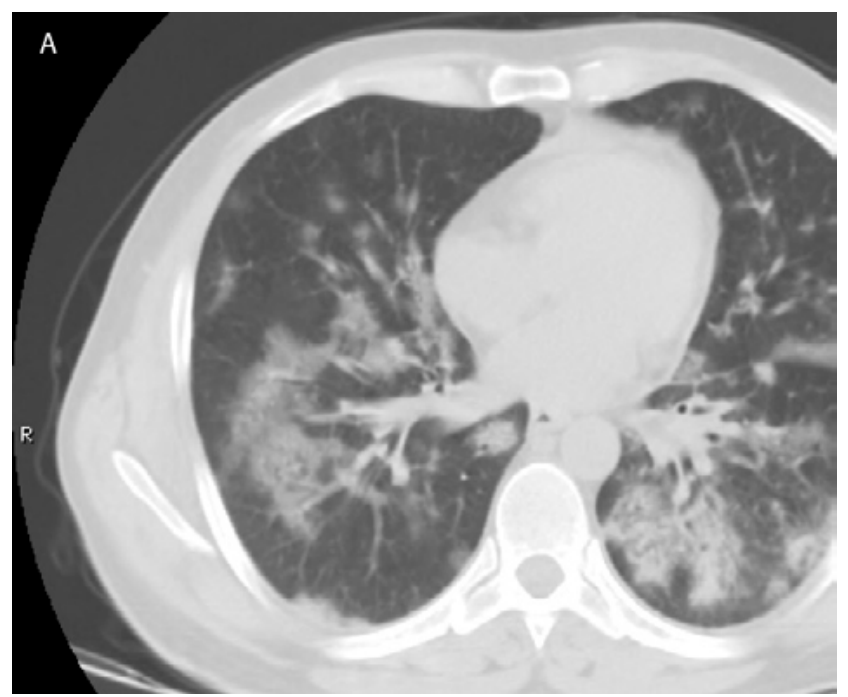

Fig. 2A and B. Chest tomography showing multifocal segmental and subsegmental consolidations with ground glass opacities and air bronchogram.

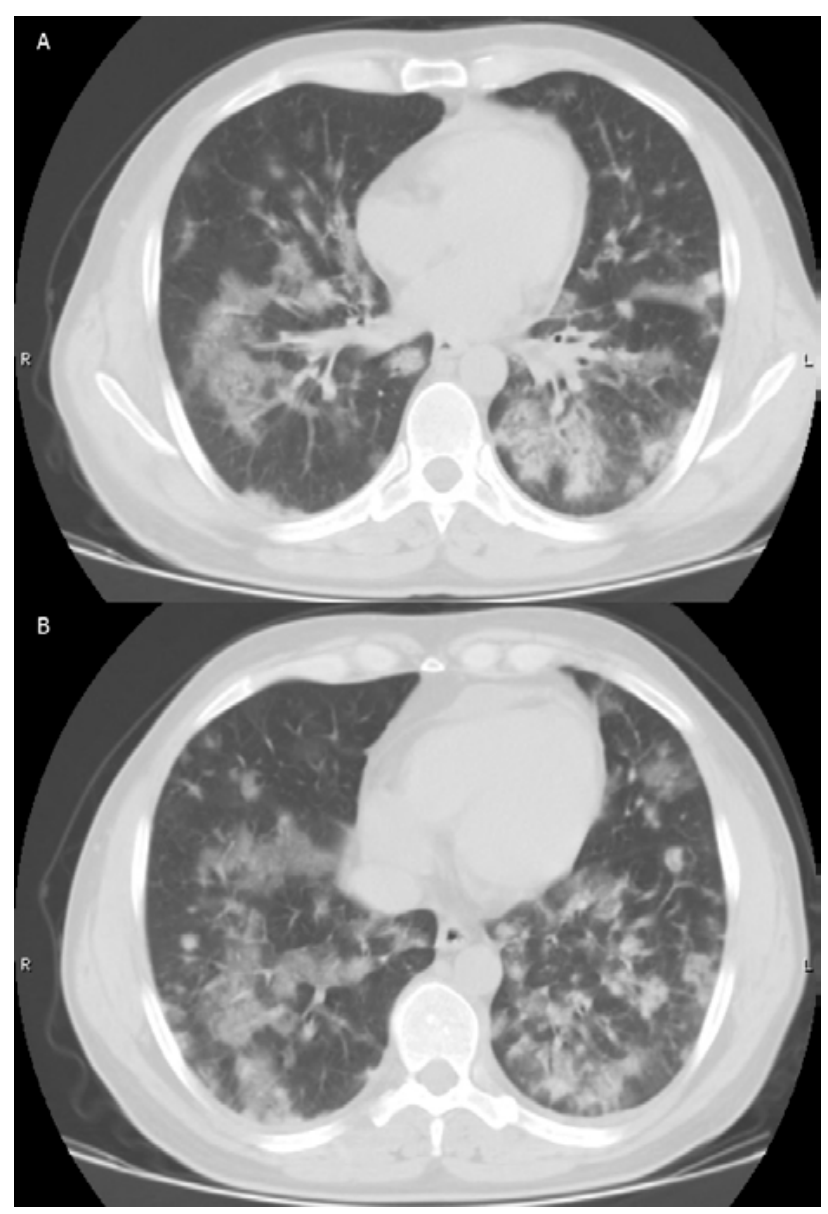

especially in the presence of ground glass or multifocal infiltrates on high-resolution tomography, without any specific difference between immunocompetent and immunocompromised patients. 3,5

As in other viral infections, spontaneous resolution may occur ${ }^{3}$, but it is consensual to treat patients who present with severe infection whenever HSV-1 is isolated from the lower respiratory tract. ${ }^{6}$ Treatment includes the use of intravenous acyclovir $5-10 \mathrm{mg} / \mathrm{kg}$ three times a day, and alternatively oral acyclovir 400-800 mg/day or valacyclovir 1000 mg three times a day.

The prognosis is related to patients' immune status, being better in the immunocompetent ones. ${ }^{2}$ Our patient remains without any sign of immunodeficiency, cancer or autoimmune disease, after 4 years of follow up.

With this case report, we aim to call attention to the importance of thinking about HSV-1 pneumonia when respiratory symptoms persist despite various antibiotic schemes, especially in the presence of ground glass or multifocal pulmonary infiltrates whose origin is unclear, regardless of patients' immune status $^{2,10}$. It is an unusual diagnosis with varied severity, possibly underdiagnosed.

\section{References}

1 Klein R: Clinical Manifestations and diagnosis of herpes simplex virus type 1 infection. In: UpToDate. Edited by Post TW, Hirsch MS, Mitty J, Waltham MA, UpToDate, 2016

2 Brodoefela H, Vogela M, Spira D, Faul C, Beck R, Claussen CD, et al. Herpes-SimplexVirus 1 pneumonia in the immunocompromised host: High-resolution CT patterns in correlation to outcome and follow-up. Eur J Radiol. 2012;81:415-420

3 Reyes CV, Bolden JR. Herpes simplex virus type-1 pneumonitis in immunocompetent young woman. Heart Lung. 2009;38:526-529

4 Pines N, Tsabari R, Kerem E, Reiter J. Herpes Simplex Virus Pneumonia in an Immunocompetent Child on Corticosteroids for Acute Wheezing. Pediatr Emerg Care. 2018;0:1-2

5 Chong S, Kim TS, Cho EY. Herpes simplex virus pneumonia: high-resolution CT findings. Br J Radiol. 2010;83:585-589

6 Miyazato A, Kishimoto H, Tamaki Z, Nakama K, Saito A. Herpes Simplex Virus Bronchopneumonia in a Non-immunocompromized Individual. Intern Med. 2001;40:836840

7 Saugel B, Jakobus J, Huber W, Hoffmann D, Holzapfel K, Protzer U, et al. Herpes simplex virus in bronchoalveolar lavage fluid of medical intensive care unit patients: Association with lung injury and outcome. J Crit Care. 2016;32:138-144

8 Bonizzoli M, Arvia R, di Valvasone S, Liotta F, Zakrzewska K, Azzi A, et al. Human herpesviruses respiratory infections in patients with acute respiratory distress (ARDS). Med Microbiol Immunol. 2016;4:371-379

9 Ishihara T, Yanagi H, Ozawa $H$, Takagi A. Severe herpes simplex virus pneumonia in an elderly, immunocompetent patient. BMJ Case Rep. 2018;07:2018

10 Jouneau S, Poineuf JS, Minjolle S, Tattevin P, Uhel F, Kerjouan M, et al. Which patients should be tested for viruses on bronchoalveolar lavage fluid? Eur J Clin Microbiol Infect Dis. 2013;32:671-677 